\title{
Familial isolated hyperCKaemia associated with a new mutation in the caveolin-3 (CAV-3) gene
}

\author{
L Merlini, I Carbone, C Capanni, P Sabatelli, S Tortorelli, F Sotgia, M P Lisanti, \\ C Bruno, C Minetti
}

J Neurol Neurosurg Psychiatry 2002;73:65-67

An 18 year old man and his mother both presented with persistent, isolated raised serum creatine kinase (hyperCKaemia) without muscle symptoms. Analysis of caveolin-3 protein expression in muscle biopsy of the propositus showed a reduction in the protein. Genetic analysis revealed a new heterozygous mutation in the caveolin-3 (CAV-3) gene: a $\mathrm{C} \rightarrow$ T transition at nucleotide position 83 in exon 1 leading to a substitution of a proline for a leucine at amino acid position 28 (P28L). This is the first pathogenic mutation in the CAV-3 gene associated with isolated familial hyperCKaemia. It expands the genetic heterogeneity in patients with caveolin-3 deficiency and confirms that caveolin-3 deficiency should be considered in the differential diagnosis of isolated hyperCKaemia.

R aised serum creatine kinase (hyperCKaemia) is a hallmark of hereditary neuromuscular disorders. ${ }^{1}$ However, several acquired conditions (for example, infections, alcoholism, drugs, intramuscular injections) may also cause a rise in creatine kinase, and as the enzyme is now often included in routine blood tests the number of apparently healthy individuals with persistent hyperCKaemia has increased. ${ }^{2}$ The criteria for the diagnosis of isolated hyperCKaemia have been revised in recent decades, with emphasis on the importance of a thorough muscle biopsy study. ${ }^{2-4}$

Caveolin-3, a muscle specific caveolin related protein, is the principal structural protein of caveolar membrane domains in skeletal muscle and in the heart. ${ }^{5}$ Mutations within the coding sequence of the human caveolin-3 gene have been so far associated with different muscle disorders: an autosomal dominant form of limb girdle muscular dystrophy (LGMD-1C), ${ }^{6}$ an idiopathic form of hyperCKaemia, ${ }^{7}$ and recently, a rare skeletal muscle disorder characterised by mechanical hyperirritability (rippling muscle disease, RMD) ${ }^{8}{ }^{9}$

We previously described a sporadic heterozygous mutation in the CAV-3 gene in two unrelated asymptomatic children with isolated persistent hyperCKaemia. ${ }^{7}$ Here, we present two members of another family with persistent hyperCKaemia without any signs or symptoms of myopathy, in whom muscle biopsy revealed partial caveolin-3 deficiency and genetic analysis identified a new mutation in the caveolin-3 gene.

\section{METHODS}

\section{Case 1}

The propositus, an 18 year old man, was referred for further evaluation of a raised serum creatine kinase discovered during a routine blood test. Motor milestones were normal. He never complained of muscle weakness, cramps, or premature fatigue, even after exercise. Neurological examination showed no muscle intolerance, signs of mechanical hyperirritability, or central nervous system impairment. There was no calf hypertrophy or contractures. Resting serum creatine kinase was
$2600 \mathrm{IU} / \mathrm{l}$ (normal value < $175 \mathrm{IU} / \mathrm{l}$ ), while other routine laboratory investigations were normal. Electromyography (EMG) and nerve conduction studies were normal. An electrocardiogram was normal. Computed tomography of the shoulder, abdominal, hip, and leg muscles was normal. The patient was not taken any drugs or receiving intramuscular injections.

\section{Case 2}

A 49 year old woman, the mother of case 1, denied any myalgia or cramps. General physical examination was normal. Neurological examination and electrophysiological studies were unremarkable. Resting serum CK was 550 IU/l. Extensive routine laboratory investigations were normal.

\section{Investigations}

In both cases, serum CK levels were raised on multiple occasions; the father and the 24 year old sister had normal values.

A muscle biopsy was obtained from the quadriceps muscle of the propositus. Routine histological and histochemical techniques, including staining for myophosphorylase, phosphofructokinase, and myoadenylate deaminase, were performed by standard methods. For immunohistochemistry and western blot analysis, muscle samples were processed as previously described. ${ }^{6}$ Genomic DNA was extracted from peripheral blood leucocytes using the standard phenol/chloroform method. The full length coding region and the intron-exon boundaries of the CAV-3 gene were screened for mutations by direct sequencing of polymerase chain reaction products using specific primers, as described. ${ }^{\circ}$

\section{RESULTS}

Muscle biopsy showed a mild increase in variability of fibre size with some hypertrophic fibres, few internal nuclei, and one necrotic fibre. On immunofluorescence microscopy, the intensity of staining for caveolin-3 at the cell surface was reduced (fig 1). On western blot analysis the caveolin-3 protein expression levels were reduced by 65\% (data not shown). Other membrane proteins involved in different forms of muscular dystrophy (dystrophin, $\alpha-, \beta-, \quad \gamma-$, and $\delta$-sarcoglycan, $\alpha 2$-laminin) had normal immunostaining patterns.

Sequence analysis of the CAV-3 gene in the propositus identified a novel heterozygous $\mathrm{C} \rightarrow \mathrm{T}$ transition at nucleotide position 83 in exon 1, leading to a substitution of a proline for a leucine at amino acid position 28 (P28L) (fig l). The mutation was confirmed in the mother by direct sequencing analysis. This mutation was not observed in 50 other patients with different myopathies or in 100 normal controls.

\section{DISCUSSION}

We describe a novel heterozygous mutation- $\mathrm{a} C \rightarrow \mathrm{T}$ transition at nucleotide position 83 in exon 1 of the CAV-3 gene in two adult family members, a young man and his mother- 

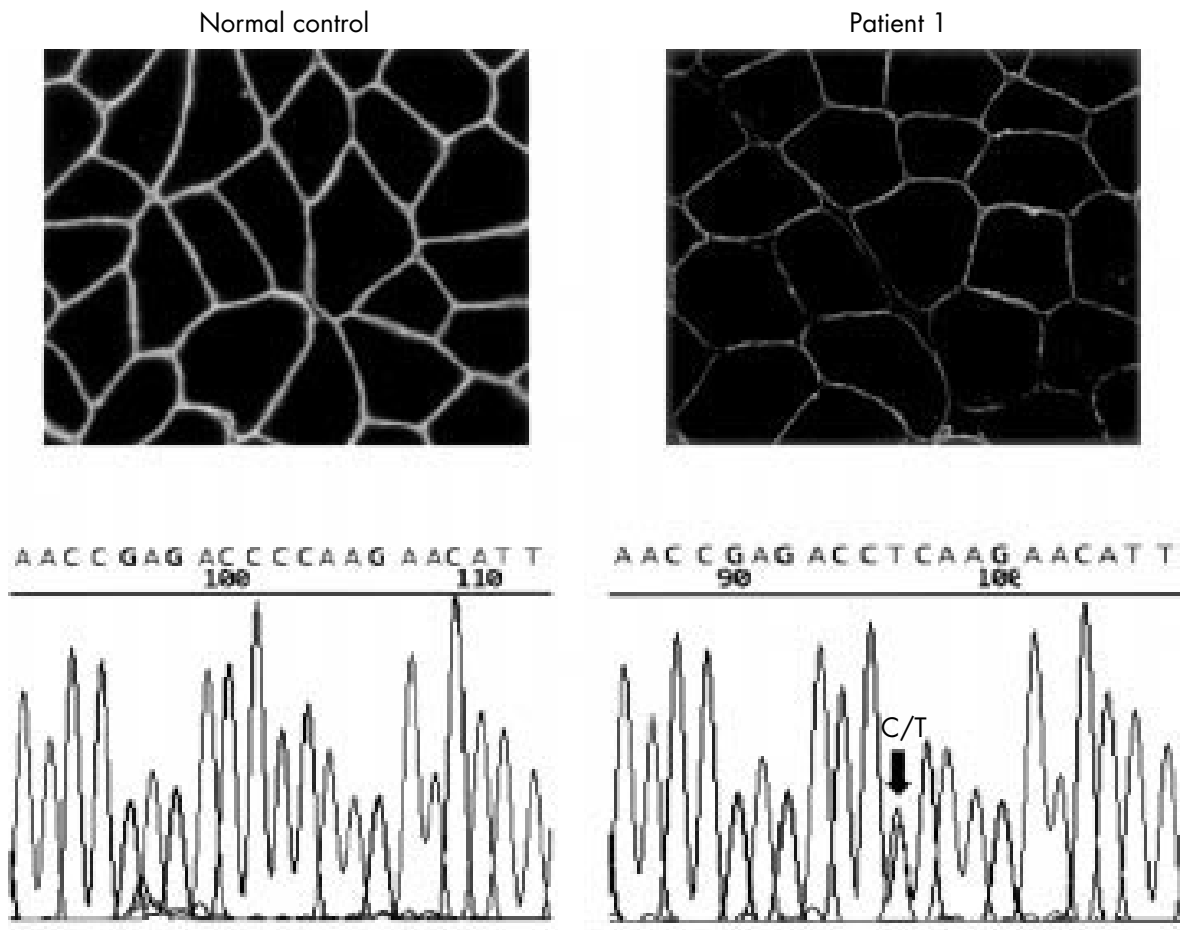

Figure 1 Upper panels: Immunolocalisation of caveolin-3 in muscle biopsy. Left: fluorescent immunohistochemical labelling of caveolin-3 in normal muscle, revealing strong positive staining at the sarcolemmal membrane. Right: immunohistochemical labelling of caveolin-3 in patient 1 showing reduced staining at the sarcolemma. Lower panels: Sequence analysis of the CAV-3 gene. Left: sequence DNA of caveolin-3 in a normal control (exon 1). Right: heterozygous $C \rightarrow T$ mutation at nucleotide position 83 (arrow) in patient 1, leading to a proline to leucine substitution (P28L).

associated with isolated persistent hyperCKaemia (three- to 15 -fold above normal values). Neither of these individuals had muscle symptoms, and we excluded the other known causes of hyperCKaemia. ${ }^{123}$ To our knowledge, this is the first report of a mutation in the CAV-3 gene associated with familial hyperCKaemia, confirming that caveolin-3 deficiency should be considered in the differential diagnosis of isolated hyperCKaemia. We believe this mutation is likely to be pathogenic, for the following reasons: first, leucine at amino acid position 28 is a highly conserved residue across evolution, suggesting a central role for this amino acid in the normal structure and function of the enzyme; second, there was co-segregatation with hyperCKaemia in a dominant mode of inheritance over two generations; third, there was cosegregatation with the reduction in the protein expression; and fourth, the anomaly was not found in any of 100 controls.

Different mutations in the CAV-3 gene have been associated with various clinical phenotypes.6789101112 We previously identified a missense mutation and a microdeletion in eight patients from two different families with a specific form of limb girdle muscular dystrophy (LGMD-1C). ${ }^{6}$ We then reported a novel sporadic mutation in two unrelated children associated only with persistent hyperCKaemia without any symptoms of myopathy. ${ }^{7}$ Recently, four mutations in the CAV-3 gene have been identified in five families with hereditary rippling muscle disorders (RMD), an autosomal dominant human disease characterised by mechanically triggered contraction of skeletal muscle. ${ }^{89}$

Interestingly, same mutations have been associated with different phenotypes, implying that other genes or factors are involved in the clinical expression (table 1). In addition, a de novo CAV-3 mutation (R26Q) localised in the $\mathrm{N}$-terminal domain was found in three different patients, ${ }^{79}$ suggesting that this region of the first exon of the CAV-3 gene may be a genetic hot spot for the development of a common sporadic mutation.

We note that the reduction of caveolin-3 protein expression observed on western blot in patients with hyperCKaemia, either sporadic or familial, is less severe than that detected in LGMD-1C. A possible explanation may be that mutations in LGMD-IC involve the scaffolding domain or the membrane

Table 1 Summary of mutations in the CAV-3 gene

\begin{tabular}{|c|c|c|c|c|c|}
\hline Mutation & Location & Base pair variation & Nucleotide position & Phenotypes & Reference \\
\hline$R 26 Q$ & EXON 1 & $\mathrm{G} \rightarrow \mathrm{A}$ & 77 & IHCK/RMD & 7,9 \\
\hline P28L & EXON 1 & $\mathrm{C} \rightarrow \mathrm{T}$ & 83 & FHCK & Present report \\
\hline A45T & EXON 2 & $\mathrm{G} \rightarrow \mathrm{A}$ & 133 & LGMD 1C/RMD & 8,11 \\
\hline A45V & EXON 2 & $\mathrm{C} \rightarrow \mathrm{T}$ & 134 & RMD & 8 \\
\hline G55S & EXON 2 & $G \rightarrow A$ & 163 & LGMD IC & 10 \\
\hline श़TFT (63-65) & EXON 2 & 9 bp deletion & $186-194$ & LGMD IC & 6 \\
\hline C7IW & EXON 2 & $C \rightarrow G$ & 213 & LGMD IC & 10 \\
\hline P104L & EXON 2 & $\mathrm{C} \rightarrow \mathrm{T}$ & 311 & LGMD 1C/RMD & 6,8 \\
\hline $\mathrm{R} 125 \mathrm{H}$ & EXON 2 & $\mathrm{G} \rightarrow \mathrm{A}$ & 374 & LGMD IC & 12 \\
\hline
\end{tabular}

bp, base pair; FHCK, familial hyperCKaemia; IHCK, isolated hyperCKaemia; LGMD, limb girdle muscular dystrophy; RMD, rippling muscle disease. 
spanning domain of caveolin-3, which are essential for caveolin homo-oligomerisation and for the formation of caveolae at the cell membrane. In contrast, a mutation involving other parts of the caveolin-3 protein, as observed in our patients with hyperCKaemia, may have less severe consequences for caveolin-3 protein expression at the plasmalemma. However, these mutations may still partially destabilise the muscle cell plasma membrane, resulting in asymptomatic release of creatine kinase.

Although the absolute number of patients examined is still too small to define the genotype-phenotype correlation, our familial cases and the two sporadic patients previously reported by us indicate that caveolin-3 deficiency may present with persistent isolated hyperCKaemia that is clinically silent.

Irrespective the clinical course of the disease, we recommend performing immunohistochemical evaluation of caveolin-3 protein expression and molecular genetic analysis of the CAV-3 gene in sporadic or familial cases of isolated persistent hyperCKaemia.

\section{ACKNOWLEDGEMENTS}

This work was supported by grants from EC project Myo-Cluster (to LM, contract No QLGl-CT-1999-00870), Telethon-Italia (to CM, project GP027/y01), and the Italian Ministry of Health (Istituto Ortopedico Rizzoli, and Istituto Giannina Gaslini, Ricerca Finalizzata).

\section{Authors' affiliations}

L Merlini, Neuromuscular Disease Unit, Istituto Ortopedico Rizzoli, Bologna, Italy

C Capanni, Laboratory of Cellular Biology and Electron Microscopy, Istituto Ortopedico Rizzoli,

P Sabatelli, Institute of Citomorphology, CNR, Bologna, Italy

M P Lisanti, Department of Molecular Pharmacology, The Albert Einstein College of Medicine, Bronx, New York, USA
I Carbone, S Tortorelli, F Sotgia, C Bruno, C Minetti, Neuromuscular Disease Unit, Department of Paediatrics, University of Genova, Gaslini Institute, Genova, Italy

Correspondence to: Dr C Minetti, Servizio Malattie Neuro-Muscolari, Dipartimento di Pediatria dell'Università di Genova, Istituto G Gaslini, Largo G Gaslini 5, 1-16147 Genova, Italy; minettic@unige.it

Received 17 January 2002

Accepted 4 March 2002

\section{REFERENCES}

1 Rowland LP, Willner J, Cerri C, et al. Approaches to the membrane theory of Duchenne muscular dystrophy. In: Angelini C, Danieli GA, Fontanari D, eds. Muscular dystrophy research: advances and new trends. Amsterdam: Excerpta Medica, 1980:3-13.

2 Afifi AK. Idiopathic hyperCKemia revisited. J Child Neurol 1998; 13:251-2.

3 Reijneveld JC, Notermans NC, Linssen WHJP, et al. HyperCKemia revisited. Neuromusc Dis 2001;11:163-4.

4 Bruno C, Bertini E, Santorelli FM, et al. HyperCKemia as the only sign of McArdle's disease in a child. J Child Neurol 2000;15:137-8.

5 Galbiati F, Razani B, Lisanti MP. Caveolae and caveolin-3 in muscular dystrophy. Trends Mol Med 2001;7:435-41.

6 Minetti C, Sotgia F, Bruno C, et al. Mutations in the caveolin-3 gene cause autosomal dominant limb-girdle muscular dystrophy. Nat Genet $1998 ; 18: 365-8$

7 Carbone I, Bruno C, Sotgia F, et al. Mutation in the CAV3 gene causes partial caveolin-3 deficiency and hyperCKemia. Neurology 2000:54:1373-6.

8 Betz RC, Shoser BGH, Kasper D, et al. Mutations in CAV3 cause mechanical hyperirritability of skeletal muscle in rippling muscle disease. Nat Genet 2001;28:218-19.

9 Vorgerd M, Ricker K, Ziemssen F, et al. A sporadic case of rippling muscle disease caused by a de novo caveolin-3 mutation. Neurology 2001:57:2273-7.

10 McNally EM, de Sa Moreira E, Duggan DJ, et al. Caveolin-3 in muscular dystrophy. Hum Mol Genet 1998;7:871-7.

11 Herrmann R, Straub V, Blank M, et al. Dissociation of the dystroglycan complex in caveolin-3-deficient limb girdle muscular dystrophy. Hum Mol Genet 2000;9:2335-40.

12 De Paula F, Vainzof M, Bernardino AL, et al. Mutations in the caveolin-3 gene: when are they pathogenic? Am J Med Genet 2001;99:303-7. 


\section{CORRESPONDENCE}

\section{Measuring carotid stenosis}

Comparing a new test with a standard involves measuring disagreement. In the case of measuring carotid artery stenosis, some of the disagreement between different tests is because of inherent differences in how the stenosis is demonstrated (test characteristics). This is what we are most interested in when assessing a new technology. However, some of the disagreement simply reflects variability in how we physically make the measurement with the standard technique. Choosing the point of maximum stenosis, choosing the point in the common carotid artery for use as a denominator, measuring from an eyepiece, or measuring from calipers all introduce variation when measuring carotid stenosis. The resulting observer variability in reporting contributes to disagreement between methods but to some extent is independent of the method used to generate the angiogram in the first place.

In the medical literature, disagreement between methods is often attributed entirely to test characteristics, with little appreciation of the role of observer variability in reporting. When one method is compared with anothe and disagreements emerge, it is not readily apparent how much of the disagreement is caused by the method used and how much by the process of measurement, unless observe variability data are also presented. In the recent paper from Patel et al, interobserver variability data are presented but their significance in relation to overall agreement does not appear to have been appreciated.

Using the data from Patel et al (tables 2 and 4) for symptomatic carotid arteries, it is noted that when 34 carotid digital subtraction angiograms (DSA) are measured by one radiologist, there was disagreement in seven cases when the same films were reported by a second radiologist. Therefore if only DSA was used, seven patients would have had "inappropriate" surgery according to which radiologist read the angiogram. This is no surprising, and such disagreement is a consistent finding in observer variability studies. $^{23}$ Observer variability in reporting DSA therefore accounted for approximately $20 \%$ of disagreement in this particular series of angiograms. This sets a limit on the maximum agreement that any alternative method can demonstrate when compared with DSA. It is clearly not reasonable to expect better agreement from another method than can be obtained by re-reporting the DSAs themselves. In Patel's table 2, when the same arteries are assessed by computed tomographic angiography (CTA) there was disagreement with DSA in seven cases, while with magnetic resonance angiography (MRA) and ultrasound there was disagreement in six and seven cases, respectively. The three alternatives thus disagree with DSA to the same extent as can be attributed to observer disagreement in reporting DSA. Put simply, the same number of missed or unnecessary operations would have occurred (roughly 20\% in this series) whatever method was used, including DSA alone. Observer variability is not confined to DSA, and the scatter plots from Patel et al (fig 2) would suggest-in keeping with other studies-that observer variability is greater for MRA and CTA than for DSA. ${ }^{1}$ It is surprising that this did not translate into more clinically important disagreements when MRA and CTA were compared with DSA. This is probably accounted for by the fact that in this study, for MRA and CTA, consensus views were taken for any disagreements greater than $10 \%$ between observers.

This highlights the important point that combining multiple observations made on the same data will reduce observer variability, and ultimately improve agreement with other methods. Partly for this reason, but also because to some extent the strengths and weaknesses of CTA, MRA, and duplex ultrasound are complementary, we would suggest that a combination of tests (we use the combination of ultrasound and MRA) should be used in preference to DSA.

What is clear from this study is that most of the disagreement between the different methods of measuring carotid stenosis can be attributed to observer variability in reporting rather than to the test characteristics of the individual methods themselves. The $10 \%$ of patients injured as a result of DSA in this study, and those who continue to be put at risk from catheter angiography in these circumstances, would be quite entitled to ask why they are exposed to a procedure which appears to offer no great advantage over safer alternatives. We suggest that more studies are not required, simply a more thorough understanding of presently available information.

G Young

Middlesbrough General Hospital, Ayresome Green Lane, Middlesbrough TS5 5AZ, UK

P Humphrey

The Walton Centre, NHS Trust, Liverpool, UK

Correspondence to: Dr G Young; gavin.young@stees.nhs.uk

\section{References}

1 Patel SG, Collie DA, Wardlaw JM, et al. Outcome, observer reliability, and patient preferences if CTA, MRA, or Doppler ultrasound were used, individually or together instead of digital subtraction angiography before carotid endarterectomy. J Neurol Neurosurg Psychiatry 2002;73:21-8.

2 Rothwell PM, Gibson RJ, Slattery J, et al. Prognostic value and reproducibility of measurements of carotid stenosis. A comparison of three methods on 1001 angiograms. European Carotid Surgery Trialists' Collaborative. Stroke 1994;25:2440-4

3 Young GR, Sandercock PA, Slattery J, et al Observer variation in the interpretation of intra-arterial angiograms and the risk of inappropriate decisions about carotid endarterectomy. I Neurol Neurosurg Psychiatry 1996;60:152-7.

\section{Author's reply}

Doctors Young and Humphrey highlight that differences between tests arise from several factors, some of which are inherent in the test and some of which arise from aspects attributable to observer variation. Some of the aspects to do with observer variation apply to interpretation of all tests and some are specific to certain tests. In our study we were endeavouring to quantify the effect on patient management if non-invasive tests were used instead of intra-arterial angiography to assess carotid stenosis. Our study has several limitations, including a relatively small sample size, and the fact that we were not able to get all scans read by all observers but rather had to get pairs of observers to concentrate on reading only CTA, or MRA, or DSA. A better design would have been to keep the same workers together in pairs but randomly assign the CTA, MRA, or DSA films to each pair. As it is, it is possible that some of the apparent difference between imaging modalities is specific to the pair of observers, not to the modality. However, imaging studies are difficult to fund and expensive to do, and the result and design of our study was a compromise involving all these factors.

We identified that the observer reliability of CT angiography or MR angiography was worse than that for digital subtraction angiography, as highlighted by Drs Young and Humphrey. Also in general there was more scatter between the observers for the reading of asymptomatic stenoses than for symptomatic stenoses (emphasising the importance of considering patient characteristics, not just the imaging technique). In the determination of the effect that this disagreement might have on patient management, we used nomograms derived from the European carotid surgery trial which were based on intra-arterial angiographic measurement of stenosis. We therefore had to use the comparison of non-invasive test reading with DSA rather than being able to use the individual observers readings of non-invasive tests. Thus as Drs Young and Humphrey point out, the actual effect of using non-invasive tests maybe worse than we have estimated.

Finally, Drs Young and Humphrey suggest that more studies are not required but we are not entirely sure that that is completely true. Non-invasive imaging tests are continually undergoing modifications, many of which may be improvements in accuracy or practicality, but this cannot be assumed to be the case. Much of this tinkering with technology is driven by the manufacturer's desire to encourage purchase of new machines. Improvements have also occurred in intraarterial angiography with smaller and more manoeuvrable catheters and greater awareness of the risks, which may have helped to reduce the risk of angiography. Our "snap shot" of CTA, MRA, and ultrasound is already out of date because contrast MRA is now increasingly used. While we would hope that non-invasive tests (probably in combination rather than alone) would eventually replace intra-arterial angiography in the majority of patients being considered for carotid intervention, we feel it likely that there will always be a need for some intra-arterial angiography in specific cases, or depending on local resources. In any case DSA did not appear less popular than MRA among the patients in our study. There is certainly room for much more in depth examination of existing data but we shouldn't close the door on the need for further studies. 


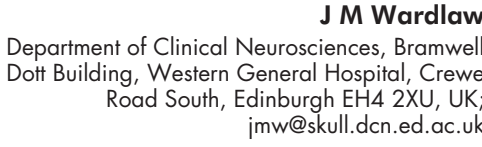

\section{Cerebral metastasis after primary renal cell carcinoma}

The article by Roser et al, ${ }^{1}$ in which it was shown that the treatment of intracranial metastases originating from renal cell carcinoma can on occasion be successful, was most interesting.

We have followed the clinical course of a patient with a renal cell carcinoma with a low mitotic index since 1989. In this patient the course was distinctly more malignant but the disease has also been successfully treated to date. In the last 13 years, this patient has had four metastases surgically removed and a further nine treated with stereotactically guided percutaneous single dose convergent beam irradiation therapy (stereotactic modified linear accelerator, 6-15 MV photons, 18-20 Gy prescribed to the $80 \%$ isodose). Apart from slight mnemonic deficits, the patient is in good health

The following factors which affect the prognosis $^{2-4}$ were all met by our patient

- The interval between the diagnosis of renal cell carcinoma and the first detected brain metastasis exceeds 17 months (our patient, 18 months; the patient described by Roser et al, ${ }^{1} 36$ months);

- Age below 60 years at the time of initial diagnosis;

- Primary tumour of the left kidney, initial nephrectomy;

- Diameter of primary metastasis $<2 \mathrm{~cm}$;

- Not more than one brain metastasis at the time of initial treatment;

- Solely intracranial metastases;

- Karnofsky > 70\%;

- No systemic symptoms such as fever or weight loss at the time of diagnosis;

- Blood sedimentation rate under $50 \mathrm{~mm} / \mathrm{h}$ at diagnosis of renal cell carcinoma.

Patients in whom prognostic factors predict a good outcome should be treated with intent to cure.

S Heckl

Department of Neuroradiology, University of Heidelberg Medical School, Kopfklinik, Im Nevenheimer Feld 400, 69120 Heidelberg,

Germany

K Braun, J Debus

Department of Radiation Oncology, German Cancer Research Centre, Heidelberg

S Kunze

Department of Neurosurgery, University of

Heidelberg

Correspondence to: Dr med Stefan Heckl s.heckl@dkfz-heidelberg.de

\section{References}

1 Roser F, Rosahl SK, Samii M. Single cerebral metastasis 3 and 19 years after primary renal cell carcinoma: case report and review of the literature. J Neurol Neurosurg Psychiatry 2002;72:257-8.

2 Decker DA, Decker VL, Herskovic A, et al. Brain metastases in patients with renal cell carcinoma, prognosis and treatment. J Clin Oncol 1984;2: 169-73.
3 Wronski MD, Maor MH, Davis BJ, et al. External radiation of brain metastases from renal carcinoma: a retrospective study of 119 patients from the M D Anderson Cancer Center. Int J Radiat Oncol Biol Phys 1997:37:753-9.

4 Culine S, Bekradda M, Kramar A, et al. Prognostic factors for survival in patients with brain metastases from renal cell carcinoma. Cancer 1998;83:2548-53.

\section{BOOK REVIEWS}

\section{Seizures, medical causes and management}

Edited by Norman Delanty (Pp 352 US\$1 25.00). Published by the Humana Press New Jersey, 2002. ISBN 0-89603-827-0

This book is unusual among books about seizures because it focuses on acute symptomatic ("situation-related") seizures, rather than "epilepsy" (although there is inevitably some overlap between the two). It provides definitions and describes the epidemiology and pathophysiology of acute symptomatic seizures in the initial section, which is followed by chapters detailing the specific circumstances in which such seizures are likely to occur, often (although not invariably) including points of management specific to the situation. Subjects covered include seizures occurring in the context of multisystem disease, infection, hypoxic-ischaemic cardiopulmonary conditions, endocrine disorders, cancer and other conditions. Situation-related seizures occurring as a result of drugs or alcohol misuse are also addressed, as are those occurring in the intensive care situation, and the difficult, but important, differentiation of seizures from syncope. The book ends with a very practical chapter entitled "Anticonvulsants in acute medical illness", in which the considerations affecting the choice of antiepileptic drug in the acute situation are reviewed.

Although situation-related seizures are usually discussed in books about epilepsy, they do appear to constitute a distinct group in a number of respects including prognosis. To a certain extent the topics discussed in the book form a rather disparate group linked only by their tendency to cause such seizures as a reflection of central nervous system disturbance. Nevertheless, they are all conditions likely to be encountered at various times by general physicians, neurologists, and those working in the accident and emergency department, and this book, which is both readable and comprehensively referenced, will be of interest to all these groups.

Yvonne Hart

\section{Subcortical stroke, 2nd edition}

Edited by Geoffrey Donnan, Bo Norrving, John Bamford, and Julien Bogousslavsky (Pp 450, £79.50). Published by Oxford University Press, New York, 2002. ISBN 0-19-263-157-8

This book is a must read for clinicians and researchers with an interest in stroke. The four editors are all specialist stroke clinicians who have been thinking about and leading research in subcortical stroke for many years, and they have put together a well constructed and comprehensive multiauthor work. This second edition is longer and more extensive than the first, reflecting the considerable and rapid advances in our understanding of subcortical strokes in recent years, and in particular the increasingly sophisticated neuroimaging techniques. Given the large number of contributors, consistency of style and approach is limited, but this is more than made up for by the breadth of expertise and opinion.

There are some particular strengths. These include the editors' short chapter providing a summary classification of subcortical infarcts, which is best appreciated if read both before and after tackling most of the other chapters. The excellent chapter on pathology of lacunar infarction is a welcome addition to this edition, while the chapters discussing risk factors and prognosis provide very useful commentaries and summary tables of all the relevant studies. The discussion around the usefulness (or not) of clinical diagnosis of lacunar syndromes, carefully updated with the information from recent clinicoradiological studies, is both thoughtful and logical, with plenty of clinical and epidemiological common sense.

In common with all recently published medical textbooks, this one is already a little out of date. This is most noticeable for the chapter on therapy, where recent advances (for example, new evidence on blood pressure lowering from the PROGRESS trial and on cholesterol reduction with a statin from the Heart Protection Study) are likely to have most impact on clinical practice. If the editors have the energy to produce a third edition, there is (as always) some room for improvement. The series of chapters on infarcts in specific subcortical territories would be enhanced by some figures illustrating the vascular anatomy that is discussed in the text. In addition, the quality of the discussion of study methodology varies considerably between chapters, and some would benefit from a more systematic and accurate approach to statistical and epidemiological concepts.

Cathie Sudlow

\section{Medical risks in epilepsy}

Edited by Svein I Johannessen, Torbjörn Tomson, Matti Sillanpää, and Birthe Pedersen (Pp 140, £29). Published by Wrightson Biomedical Publishing Ltd, Hampshire, 2002. ISBN 1- 871816-46-7

This is a very useful, reasonably comprehensive yet succinct multiauthor small book on medical risks associated with epilepsy. Areas covered include methodological aspects; accidents and risks in everyday life; traffic accidents; driving regulations; mortality, including SUDEP; psychiatric comorbidity and suicide; fatal adverse drug reactions reporting data (which are rather difficult to interpret); seizure-warning systems and risk prevention; as well as insurance related issues. It also highlights many areas where further research is required. The book generally provides an overview of the more recent research and publications in this area and includes some regulatory issues. Inevitably it has a Nordic emphasis; it includes very useful advice on precautionary measures to minimise risk of injury for people with uncontrolled epilepsy, including safer sauna. Some chapters, by necessity, serve purely as a compilation of available incomplete data. Others are written by key researchers directly involved in the area addressed and provide a very balanced review of current knowledge. On psychiatric comorbidity, while agreeing that "the positive 
effects of drug therapy on cognitive and affective functioning because of the reduction in seizure activity are usually far greater than the negative effects", more information would have been welcome in an otherwise very well balanced chapter. The book would well serve those for whom it is intended, namely epileptologists, neurologists, paediatric neurologists, psychiatrists, and other professionals who deal with patients with epilepsy. The editors rightly stress the "official line" that the majority of patients with epilepsy can achieve good control, with low associated risks.

Lina Nashef

\section{Greenfield's neuropathology, 7th edition}

Edited by David I Graham and Peter L Lantos. (2 Vol set (HB), Vol 1 ppl 190, Vol 2 ppl 140 $£ 395)$. Published by Arnold, London, 2002. ISBN 0-340-74231-3. CD rom (£145) ISBN 0-340-76-221-7.

What can one say. The latest (7th) edition of Greenfield's Neuropathology has hit the bookshops, and indeed what a resounding thud it makes! The present edition is bigger than ever, again running into two volumes, but now totalling a staggering 2330 pages and costing an equally staggering $£ 395$. It comes equipped with a handy $C D$ version of the illustrations, a mere snip at $£ 145$.

The 7th edition has undergone considerable changes in content, since the last edition five years ago, reflecting the ever expanding increase in knowledge of diseases of the nervous system and muscle that has come from the exponential growth in neuroscience research over the past decade. Areas of cellular and molecular neurobiology, and the contributions that genetics and neuroimaging have made towards improving our understanding of the causes of disease and our clinical investigative and diagnostic skills, are more strongly featured. Hence, while greater emphasis has been placed on the basic science of disease, the classic descriptive morphology for which Greenfield's is renown is well maintained. There are new chapters on "Metabolic and neurodegenerative diseases of childhood" and "Peroxisomal and mitochondrial diseases". The chapter on "Pathology of schizophrenia" has been shrewdly expanded to cover "The pathology of psychiatric disorders". Other chapters have been retained as such, but many have been rewritten with new authors reflecting the pre-eminence of each within their particular subspecialty. There is increased reliance on colour illustrations, line diagrams and tables to illuminate the text and these are of excellent quality throughout. As to be expected, all chapters are written authoritatively with clarity and style, comprehensively illustrated, and lavishly referenced. Judging by the content of the chapters on ageing and dementia, prion disease, and movement disorders, it is my guess that if anything is not included in each chapter, it's probably not worth including anyway. The accompanying $\mathrm{CD}$ rom is user friendly, and the images are downloadable - $\mathrm{a}$ boon to those wishing to produce a ready made lecture or presentation of distinction. The book is a must for practicing and trainee pathologists, but is equally compelling for workers in other clinical neuroscience disciplines and basic researchers interested in the roots of the dysfunctional nervous system. Possession of the 7th edition is guaranteed lasting quality and full value, but before lashing out make sure both your arms and shelving are strong enough to accommodate its presence.

David MA Mann

\section{Smell and taste complaints}

Edited by Christopher H Hawkes (Pp 176, £16.99). Published by ButterworthHeinemann, Woburn, 2002. ISBN 0-75067287-0.

Despite the fact that problems with tasting and smelling are common in the general population, few physicians have the knowledge and training to authoritatively deal with them. Christopher Hawke's Smell and Taste Complaints provides a straightforward guide to the understanding and management of chemosensory disturbances, reflecting the first clinically oriented book of its kind since Ellis Douek's The Sense of Smell and its Abnormalities (Edinburgh: Churchill Livingstone, 1974). This 180 page pocket sized book provides a cogent overview of the anatomy and physiology of the olfactory and gustatory systems, practical approaches towards their assessment, and suggestions for therapy and management. Importantly, it provides the practitioner with the names and addresses of specialised taste and smell clinics throughout the world, aiding the referral process. Although there is little new in this guide, and much of the material seems to have been derived from second hand sources, it presents the available information in a well organised and easy to read manner. Moreover, it addresses basic clinical issues rarely addressed in a single publication. Its major drawback is the lack of reference backing for many of its statements, some of which are questionable. I found, for example, some of the "facts" unfamiliar, and would have welcomed knowledge of their source. Bits of the material are dated (for example, the role of $\mathrm{IP}_{3}$ receptors in olfactory function, the nature of olfactory receptor cell regeneration) and several sections of the book seem lengthy, uncritical, and of little practical value. Thus, nearly seven pages are devoted to the topic of odour memory, a topic with inherent theoretical issues and problems that are not addressed by the author. However, the book is not intended to be a research book and, despite such shortcomings, it accomplishes its goal of educating the practitioner and providing him or her with a practical roadmap for clinical assessment and treatment. Indeed, the clinical information provided is comprehensive and well illustrated. This inexpensive book is a must for any physician who has the occasion to see patients with chemosensory disturbances or has even a casual interest in chemosensation, and should serve to elevate the level of appreciation of these senses within the medical community at large.

Richard L Doty

\section{CORRECTIONS}

The following errors occurred in the short report by Merlini L, Carbone I, Capanni C, et al. Familial isolated hyperCKaemia associated with a new mutation in the caveolin-3 (CAV-3) gene. J Neurol Neurosurg Psychiatry 2002;73:65-7. On page 66, left column, line 9, proline should replace leucine, line 12, protein should replace enzyme, and in table 1 , line 8 Del-TFT (63-65) should replace ?TFT (63-65).

We regret that an editing error occurred in the correspondence from Jaster JH, Dohan FC, and $\mathrm{O}^{\prime}$ Brien TF. Demyelination in the brain as a paraneoplastic disorder: candidates include some cases of seminoma and central nervous system lymphoma. J Neurol Neurosurg Psychiatry 2002;73;352. The description of a patient was altered, in the first line of the fourth paragraph the text should read "... patient who had a non-neurological malignancy, seminoma, and subsequently developed a paraneoplastic syndrome ...". 